\title{
Microstructured Reactors Designed by Stereolithography and Characterized by Fluorescent Probes
}

\author{
S. Corbel, ${ }^{1}$ F. Evenou, ${ }^{1}$ F. Baros, ${ }^{1}$ N. Martinet, ${ }^{2}$ M. Donner, ${ }^{2}$ and M. C. Carré ${ }^{1}$ \\ ${ }^{1}$ Département de Chimie Physique des Réactions, UMR 7630 CNRS-INPL, Nancy Université, 1 Rue Grandville, \\ BP 20451, 54001 Nancy Cedex, France \\ ${ }^{2}$ Centre de Ressources Biologiques, INSERM U724, Avenue de la Forêt de Haye, 54505 Vandoeuvre les Nancy, France
}

Correspondence should be addressed to S. Corbel, serge.corbel@ensic.inpl-nancy.fr

Received 4 April 2008; Accepted 30 May 2008

Recommended by Mohamed Sabry Abdel-Mottaleb

\begin{abstract}
The main objective of this research was to define a structured and functionalized support for future biomedical applications (model of "low-density bioarray"). The experiments were carried out by using stereolithography process with a special SU-8 photoresist and the reproducibility of the method was studied by analyzing the surface profile of the support. Finally, a matrix of regular controlled sized wells was fabricated. Chemical reactions leading to covalent grafting were run to demonstrate that the inner surface of the wells remains still reactive after polymerization. The grafting of fluorophores with carboxylic functions activated by $\mathrm{N}$-hydroxysuccinimide was studied as function of time, in order to determine the best reactions, conditions. Then, the grafting of two distinct fluorescent probes was led simultaneously inside the wells, showing the possibility of spatial localization of diverse reactions on the same support. The covalent and localized bindings were confirmed by fluorescence spectroscopy and microscopy analyses.
\end{abstract}

Copyright $\odot 2008$ S. Corbel et al. This is an open access article distributed under the Creative Commons Attribution License, which permits unrestricted use, distribution, and reproduction in any medium, provided the original work is properly cited.

\section{INTRODUCTION}

Probes biomolecules arrays are nowadays more and more used in research field to detect, determine, or quantify complementary targeted biomolecules (DNA, proteins, cells, tissues, etc.). An important point is the probe biomolecule immobilisation on the support which must be well located, stable (a covalent binding with the support is suitable) while keeping binding properties of biomolecules. Among the different technology processes for reaching these structured biosensor supports, thin polymer film-based substrates are currently used to afford robust patterns [1-5]. So, the shape of the polymeric structure was devised to fit the "tools" used in Life Sciences Laboratories: multichannel micropipets for multiple simultaneous depositions, microplates readers for analyses.

In this paper, we demonstrate that photolithography remains a simple and reproducible process for polymer patterning that generates surface-confined amino groups which can be subsequently used for immobilisation of compounds. Through the use of stereolithography process, we have fabricated a polymeric matrix including millimetresized wells on the surface of glass slides.

Thanks to the hydrophobic nature of the polymer, the ministructures so made can also provide ideal 3D aqueous environment which might be very convenient for the capture of biomolecules.

The work can be resumed in three steps:

(1) the stereolithography process to get the polymeric matrix,

(2) the covalent grafting of fluorescent probes to demonstrate that the surface remains reactive in the wells and finally,

(3) the fluorescence to validate the grafting.

\section{LOCALIZATION OF REACTIVE SITES ON A MICROSCOPE SLIDE BY STEREOLITHOGRAPHY}

\subsection{The supports and products}

The supports, glass microscope slides silanised with an amino linker, 3-aminopropyltriethoxysilane (APTS), chemicals, 
and solvents were purchased from Sigma-Aldrich (Chimie Tech Services, Anthony, France). After having tested different monomers, SU-8 photoresist (MicroChem Corp., Newton, Ma USA) was selected. Its formulation is based of EPON SU8 resin (glycidyl-ether-bisphenol-A novolac) dissolved in $\gamma$ butyrolactone ( $52 \mathrm{wt} \%$ ), and a triaryl sulfonium salt as the photoinitiator. The main interesting physical characteristics are the low density $(d=1.164)$ which allows an easy recoating and the high glass temperature $\left(T_{\mathrm{g}}>200^{\circ} \mathrm{C}\right)$ suitable for thermal treatment.

Stability of the cured resin was tested by immersing coated slide in different solvents at room temperature. The polymer appeared very stable in solvents such as methylene chloride, absolute ethanol, and acetone (2 days for each of them) in propylene glycol methyl ether acetate (PGMEA), sodium bicarbonate buffer $\mathrm{pH} 8.5$ (5 days). In pure DMF, the film was completely and rapidly stripped from the surface keeping its patterned structure.

After the demonstration that polymer issued from SU-8 was sufficiently resistant, we investigated the step of shaping by polymerization.

\subsection{The coating and photopolymerization}

The process comprised the coating, curing, and baking operations. Polymerization occurred on one side of the slide, except on the frosted end. Prior to use, silanized glass slides were rinsed with acetone and dried. SU-8 photoresist $(2 \mathrm{~mL})$ was dropped on the centre of each slide and spun on its surface using a spin coater (Karl Süss, RC Spin Coater CT62 V08, Saint Jeoire, France) at a spin speed of $600 \mathrm{rpm}$ during 30 seconds. Solvent was evaporated by a baking on a hot plate at $95^{\circ} \mathrm{C}$ during 5 minutes. Higher baking times were found to lead to air bubbles formation inside SU-8 film.

Stereolithography process was used to polymerize photoresist at the surface of the slide (Figure 1). This technology enables the fabrication of many complex shapes and has been presented in previous papers [6]. The Spectra-Physics Model 2020 argon ion laser was used for irradiations in the near ultraviolet (wavelength $363.8 \mathrm{~nm}$ ) with an exposure of $300 \mathrm{~mJ} / \mathrm{cm}^{2}$. Matrix of regular polymeric wells was obtained by successive duplications of an empty disc pattern inside a full polymer square, in the four directions (right, left, up, down). The shape is composed of a basic element like a hollow cylinder surrounded by a filled surface around it (Figure 2).

A program has been written in $\mathrm{C}++$ for tracing the pattern with galvanometric mirrors directly on the basis of geometric parameters, without going through the usual steps of CAD, slicing, and manufacturing. To get compact cross-linking, we performed the hatch spacing up to a value less than the laser beam diameter, in the two orthogonal directions $(x, y)$. The spot laser diameter was $0.2 \mathrm{~mm}$ in width, while the hatch space was set at $0.1 \mathrm{~mm}$. When drawing the shape, the software uses two kinds of vectors. The first ones are used for tracing circles and the others are used for filling the outside surface of the circle with horizontal $(x)$ and vertical $(y)$ lines called hatching.

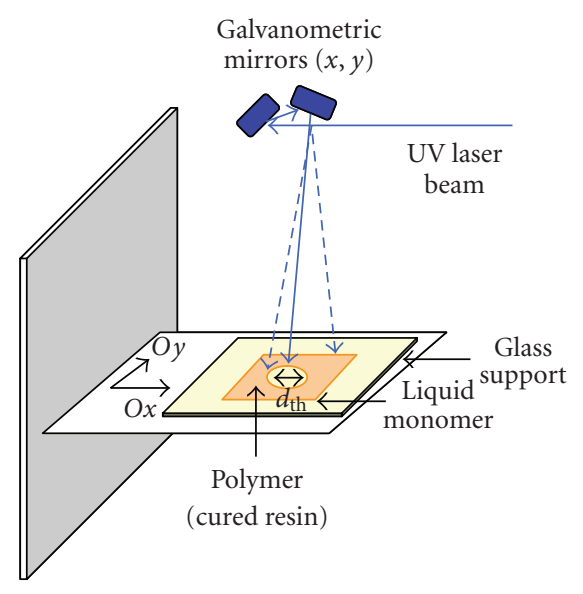

FIGURE 1: Structuration of the support by stereolithography.

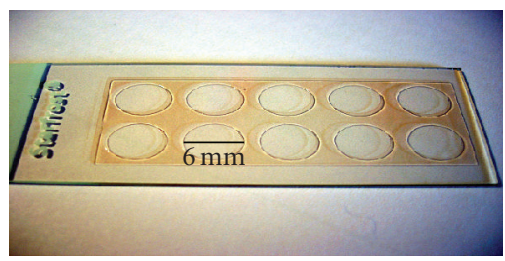

FIGURE 2: Shape of the polymeric structure obtained on the support (model of "low-density bioarray").

The improvement consisted of taking into account each point of coordinates within or outside the circle. Furthermore, there was a possibility to store hatch information in a file in order to verify the good accuracy in particular to determine whether there is an intersection between the hatching line ( $x$ or $y$ ) and contour lines as circles. Therefore, there was a double exposition at the intersection of circles and hatching lines as also at the intersections of horizontal $(x)$ and vertical $(y)$ lines of the hatching step.

In order to achieve the cross-linking of the exposed parts, a postexposure bake was carried out on a hot plate at $95^{\circ} \mathrm{C}$ during 2 minutes and then the slide was immersed in SU8 developer under agitation for about 5 minutes, then had been dried. Finally, a strong adhesion of the polymer on the surface of the slide was obtained by a postcuring under UV lamps for 15 minutes and a baking on a hot plate at $95^{\circ} \mathrm{C}$ for 30 minutes.

\subsection{Dimension characteristics of the polymeric shapes and accuracy}

Surface profiles measurements have been recorded with the optical microfocus measurement system (UBM) and actual heights of the SU-8 wells measured by scanning at the surface of the polymeric structure build on the support.

First, we defined the elementary shape of a cross-section, which is a circle of $13 \mathrm{~mm}$ diameter centred in a square of $17 \mathrm{~mm}$ side. By duplicating this pattern once, we obtained two identical wells as represented on Figure 3. Thus, to define 


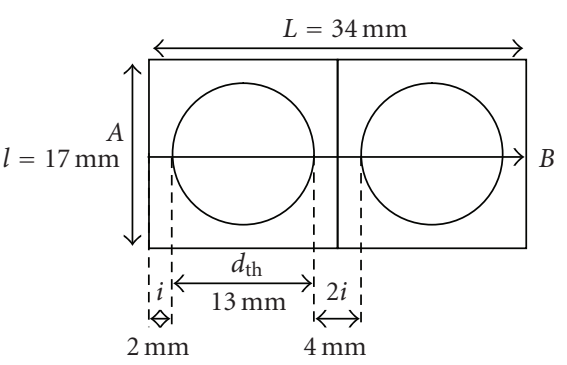

(a)

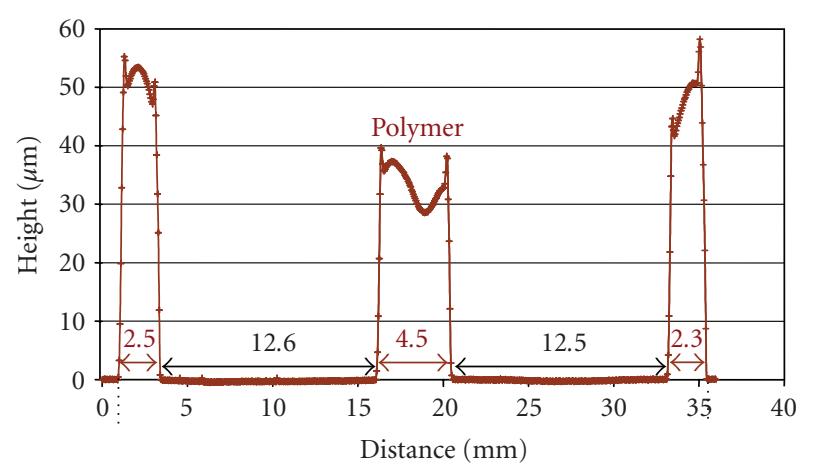

(b)

Figure 3: (a) Top view of the geometrical shape of two large wells. (b) Experimental $A B$ line profile $\left(d_{\mathrm{th}}=13 \mathrm{~mm}\right)$.

the diameter of circle equal to $13 \mathrm{~mm}$, we have to take into account the diameter of the beam $(200 \mu \mathrm{m})$.

The wells so defined and fabricated have been analyzed by the optical profilometer along the direction $A B$ as shown on Figure 3.

The line profile represents the thickness of the polymer along the line $A B$ crossing the centres of the 2 wells. The origin of the vertical axis $z$ which defines the height corresponds to the level of the horizontal plane of the glass support $(z=0)$.

We can observe that the patterns are clean and correctly reproduced. The polymeric layer seems uniform with a relatively constant thickness, except on the edge of the square where the polymer is thicker.

These small peaks due to an over polymerization may be explained, as mentioned before, by the scanning of the pattern and therefore by a supplementary curing on the borders of the shape. It can be also mentioned that the surface of the polymer is not perfectly plane. Nevertheless, the thickness of the polymer remained more or less constant (between 31 and $38 \mu \mathrm{m}$ ) with a variation which can be estimated to $18 \%$.

We have searched in a second step to manufacture smaller wells, by decreasing the diameter regularly as far as reaching a minimum value. Although the chosen resin allows the manufacturing of micro structures, the process is limited by the focus diameter of the laser beam. We kept the basic pattern which was a circle centred inside a square. The shape was duplicated by successive scanning according to one direction; we imposed a decreasing diameter from 6 to $1 \mathrm{~mm}$,

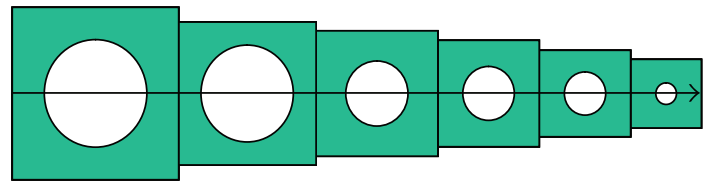

(a)

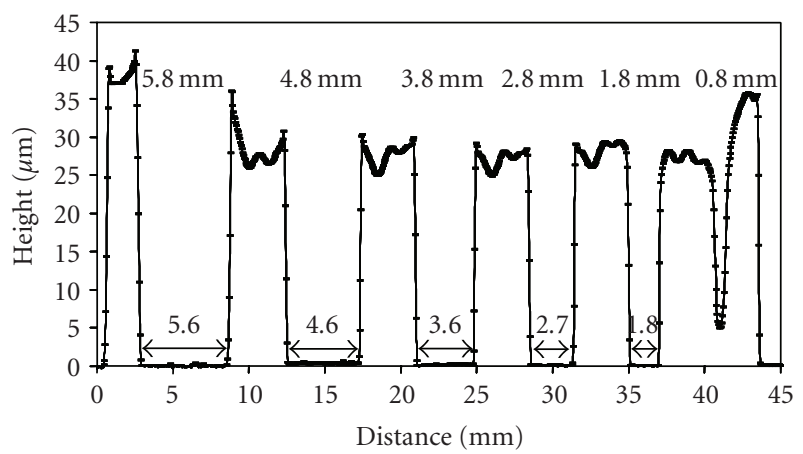

(b)

Figure 4: (a) Top view of the theoretical shape of linear matrix with decreasing diameter; (b) line profile of 6 polymer wells with decreasing diameter from 6 to $1 \mathrm{~mm}$.

while maintaining constant the interval between the circle and the outside square (Figure 4 ). The analysis of the profile, concerning a surface measuring $45 \times 12 \mathrm{~mm}$, is represented on Figure 4. Whatever the size of the well, the shape was correctly reproduced.

First of all, we notice that it is possible to manufacture wells until $1.8 \mathrm{~mm}$ of theoretical diameter presenting satisfactory lateral profiles. Indeed, it was difficult to clean the inside of the smallest wells (around $1 \mathrm{~mm}$ ). Between the wells, the polymer walls have a height of $27 \mu \mathrm{m}$ on average. As it has been observed previously, the polymer was thicker on the sides with a value varying between 36 and $42 \mu \mathrm{m}$. We also found the small peaks of over polymerization affecting the outside limit of each well corresponding to the intersections of the hatching lines.

The above results imply the feasibility of more complex patterns. For example, Figure 5 shows the fabrication of a 2D matrix with a constant diameter of the wells and the resulting profile. The fabrication was performed by duplicating several times the same elementary shape according to $x$ and $y$ directions. The surface analysis was made along lines $L_{1}$ and $L_{2}$ crossing the centres of the wells as shown on Figure 5.

One recovers the peaks of over polymerization already mentioned. Far from the edges, the polymer surface remains constant according to one axis. However, the polymer is globally thicker in $L_{2}$ than in $L_{1}$ due to the angle of the laser beam with the vertical axis in the scanning system and the consequent different irradiation intensity.

In order to determine the accuracy we have scanned a grid in polymer $(45 \times 18 \mathrm{~mm})$ in the two orthogonal directions with hatch spacing of $1 \mathrm{~mm}$. The corresponding line profile is presented on Figure 6. At first sight, we can observe that the polymer thickness is practically constant 


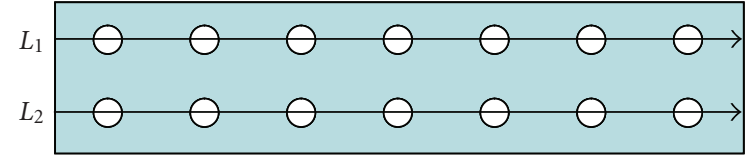

(a)

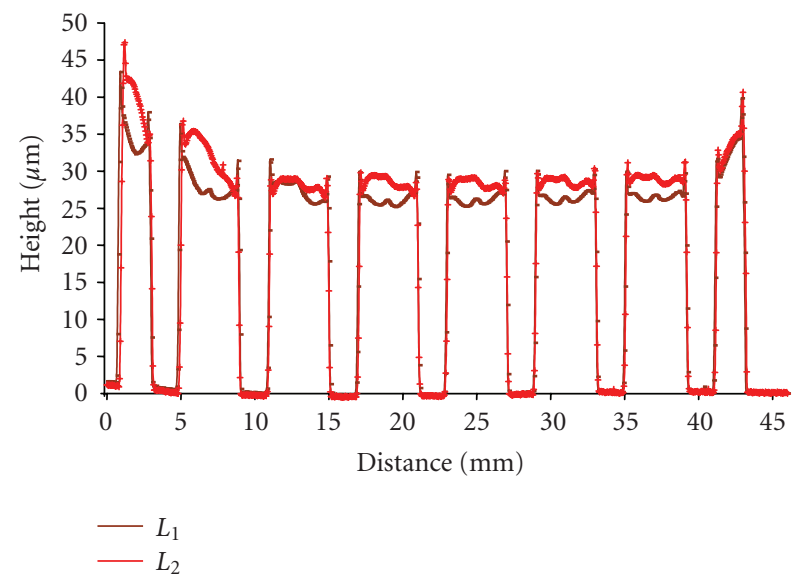

(b)

Figure 5: (a) Top view of the theoretical matrix surface with the directions $L_{1}$ and $L_{2}$; (b) surface profile along $L_{1}$ and $L_{2}$.

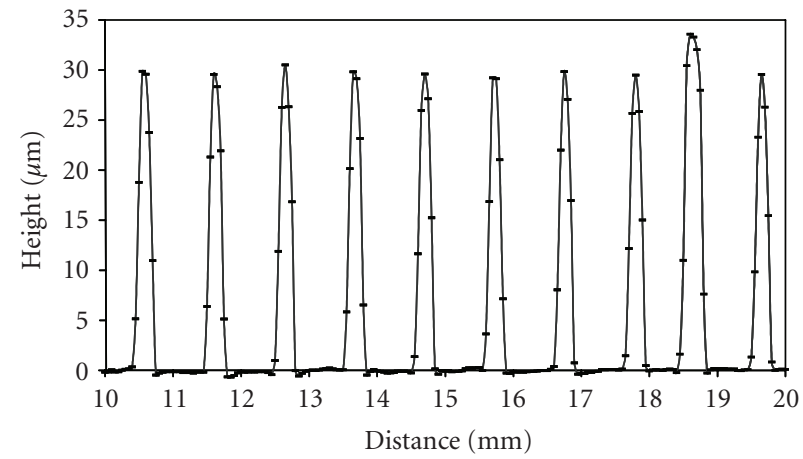

FIGURE 6: Line profile of a polymeric matrix with a $1 \mathrm{~mm}$ hatch spacing.

and equal to $30 \mu \mathrm{m}$ on the whole length. These results are otherwise very encouraging since we could define small square wells of $1 \mathrm{~mm}$ side delimited by thin walls in polymer.

\section{FLUOROPHORES COVALENT GRAFTING AND CHARACTERIZATION}

Glass surfaces are well adapted for microarray applications because they present good mechanical properties, with low intrinsic fluorescence. They also possess a relatively homogeneous chemical surface which can be easily modified and furthermore, they are not very expensive. For our study, we have selected commercially available microscope slides already silanized by a hetero linker bearing an amino terminal function.<smiles>CN(C)c1ccc(/C=C/C=C(\C#N)C(=O)O)cc1</smiles>

(a)<smiles>CCN(CC)c1ccc2cc(C(=O)O)c(=O)oc2c1</smiles>

(b)
Figure 7: Structure formulas of the two fluorophores $\mathrm{CiCOOH}$ and $\mathrm{CouCOOH}$.

Furthermore, for a future sensor application, the spots need to be uniform, accurate without spreading the analytes solution to the adjacent areas of the macroarrays. We have thought that these requirements could be achieved through the creation of wells embedded in a polymer covering the surface.

\subsection{Fluorophores covalent grafting}

Chemical reactions leading to covalent grafting were run to demonstrate that the inner surface of the wells remains active after the polymerization.

We have chosen as models two distinct wavelength fluorescent probes already studied in our laboratory [7, 8]. These fluorophores are "molecular rotors" easily synthezised: the first compound 2-cyano-5-(4-(N,N-dimethylamino)phenyl)-2,4-pentadienoic acid (CiCOOH) is a cinnamilydene derivative [7]; the second one, 7-(N,Ndiethylamino) coumarin-3-carboxylic acid ( $\mathrm{CouCOOH})$, has a coumarin structure [9] (Figure 7). The two fluorescent probes comprise in their structure a carboxylic acid function well suited for a covalent robust amide bond with the amino of the surface.

As previously described about covalent graftings of other acidic compounds on solid substrates [10], the acidic function has been activated with N-hydroxysuccinimide.

Grafting of fluorophore active esters to amino solid support through amide bond, occurs at room temperature, safe from light, in sodium bicarbonate buffer, $\mathrm{pH}$ 8.5. After grafting, the slides were carefully washed by successive immersions under magnetic stirring, in deionised water, absolute ethanol, and dichloromethane. Then, they were air dried and stored until fluorescence analysis. The covalent bindings on the slides were confirmed by fluorescence spectroscopy and microscopy analyses.

\subsection{Fluorescence spectroscopy study of the grafted probes}

The covalent binding of each fluorophore was first conducted on a separate slide to define their respective fluorescence properties. For this study, the slide was immerged in the sodium bicarbonate buffer, $\mathrm{pH}$ 8.5, containing the fluorophore, under slight agitation during 4 hours. After the general work-up described above, the fluorescence has been measured at different sites all along the slides. The 
fluorescence spectra were recorded on a Horiba JobinYvon Fluorolog3 spectrofluorimeter and were corrected from lamp variations. Experiments were carried out at room temperature, the glass slide being in front-face position in order to avoid light reflection. The excitation/emission fluorescence fields of the two fluorophores were well separated as expected considering previous studies. The conditions of excitation-emission were 470-565 nm and 410-467nm for Fluorophores CiCONHX and CouCONHX, respectively.

We have gathered (Figures 8 and 9) the emission spectra obtained with each fluorophore. For the cinnamylidene derivative ( $\mathrm{CiCOOH}$ grafting), the fluorescence signal $A_{i}$ was recorded at six places on the slide (Figure 8 ). The signal $B$ (background) of the native slide has been also reported; it has been subtracted from signal $A_{1}$ to exhibit the grafted cinnamilydene fluorescence $\left(A_{1}-B\right)$; so the maximum fluorescence is well characterized at $565 \mathrm{~nm}$ for a $470 \mathrm{~nm}$ excitation. Two remarks might here be done: on the one hand, the signal is not uniformall over the slide, with a $28 \%$ variation observed between two extreme measures: this fact may be imputed on the inhomogeneity of the native slide, but on the other hand the fluorophore response is distinct from the support one at this wavelength excitation.

For the coumarine derivative (CouCOOH grafting) the fluorescence signal was also recorded at six places of the slide (Figure 9). We have not reported the signal of the native slide which was negligible at this wavelength excitation. In this case, we can see a low deviation of the fluorescence signal; indeed, a difference of $11 \%$ between the two extreme spectra has been observed. Another interesting feature is the very important observed fluorescent signal.

These two fluorophores show specific fluorescent characteristics which have been useful for the characterization of localized graftings.

\subsection{Fluorescence microscopy study for the localization of the grafted probes}

In order to determine the optimal reactions' conditions on the structured support, the grafting of these two fluorophores was studied in function of time (Figure 10). Grafting reaction was carried out by successive deposition of the same volume of known fluorophore concentration solution into the macro wells in a saturated sodium bicarbonate buffer atmosphere. The reaction occurs with time, the first deposition corresponding to the longest-time reaction and the last one to the shortest-time reaction.

The fluorescence intensities in macrowells were measured with a Zeiss Axiotech epifluorescence microscope, coupled to a Hamamatsu IP28 photomultiplier. Two filter sets were used in order to differentiate the two fluorescent probes $\mathrm{CiCOOH}$ and $\mathrm{CouCOOH}$. All measurements were repeated five times, at different places of the well, the diameter of the excitation spot being fixed to $200 \mu \mathrm{m}$. As we can see on Figure 10, the best conditions of grafting the two fluorophores were obtained after 24 hours of reaction.

Then, the grafting of the two distinct fluorescent probes $(\mathrm{CiCOOH})$ and $(\mathrm{CouCOOH})$ was led simultaneously inside the wells. For this, we have deposited the two probes

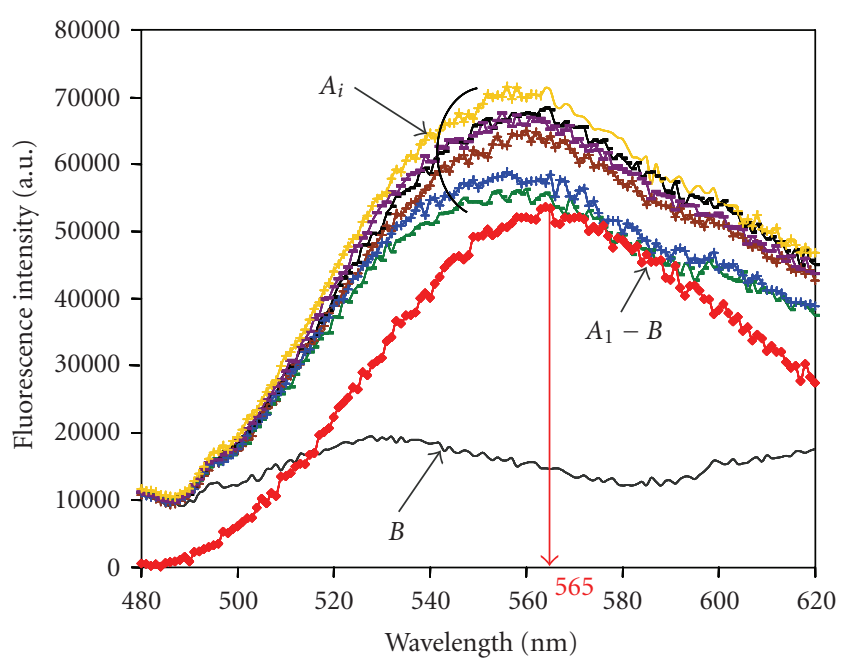

FIgURE 8: Emission spectra obtained with the cinnamylidene derivative (CiCOOH grafting); $A_{i}$ : recorded at 6 different positions on the slide; $B$ : background signal of the original slide; $A_{1}-B$ : signal corrected from background.

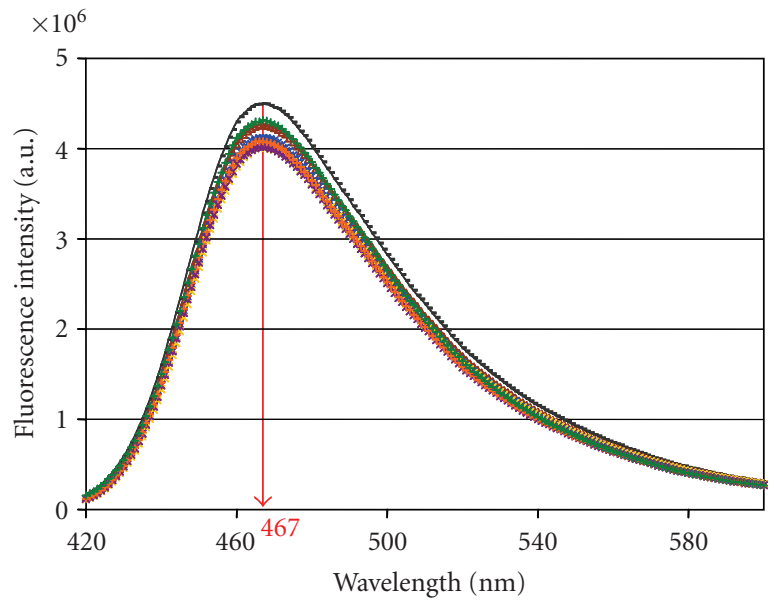

FIGURE 9: Emission spectra obtained with the coumarine derivative (CouCOOH grafting).

alternatively in a matrix of 10 wells to confirm that the inner area was still reactive after the polymerization step (Figure 11). The aim of this experiment was to prove that many different reactions may be conducted on a same support which is a condition of future developments in the industry for diagnostics test.

The fluorescence of each probe was recorded in five selected places in the same well, by fluorescence microscopy. Dispersion of measurements is shown on Figure 12 using the filters set relative to $\mathrm{CouCOOH}$ probe. Even if homogeneity in a well seems not as good as those obtained on a bare slide, due to the smaller size of the observation spot, the two probes are clearly differentiated, in spite of a weak overlap between the two filter sets. 


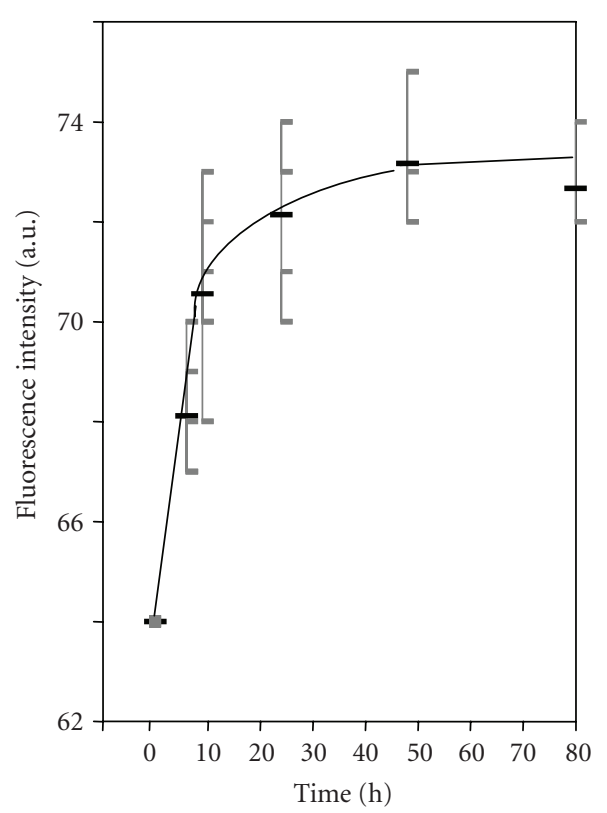

(a)

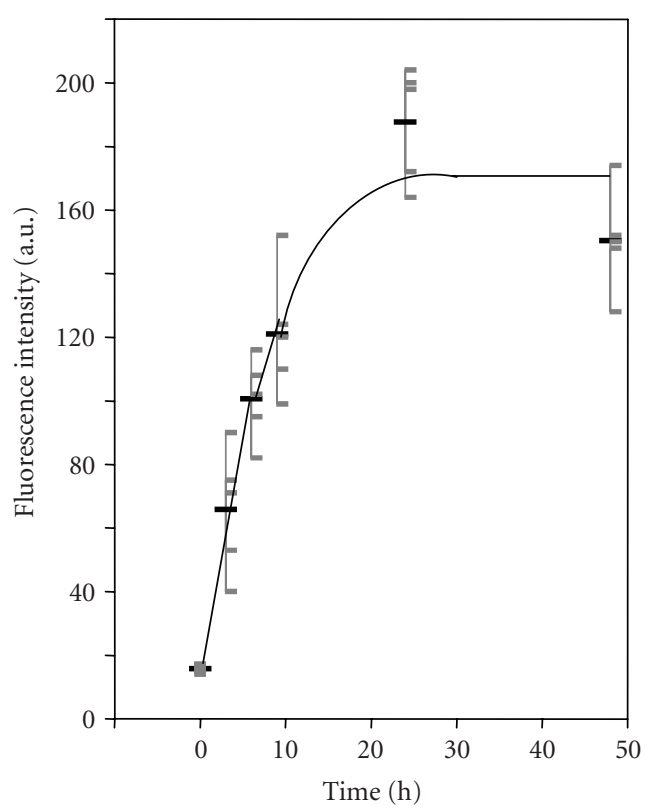

(b)

FIgURE 10: Time evolution in covalent grafting of CiCOONHS ((a) 6 hours, 9 hours, 24 hours, 48 hours, and 80 hours) and CouCOONHS ((b) 3 hours, 6 hours, 9 hours, 24 hours, 48 hours). The best conditions were reached after 24 hours of reaction.

\section{CONCLUSIONS AND FUTURE WORKS}

This preliminary work validates the fabrication process for generating polymeric matrix of wells, the size, the geometry, the number, the localization of which being well controlled. We have demonstrated the possibility to localise reactive sites in a polymer matrix shaped on a commercial glass slide. In this way, three main points have been developed. The local

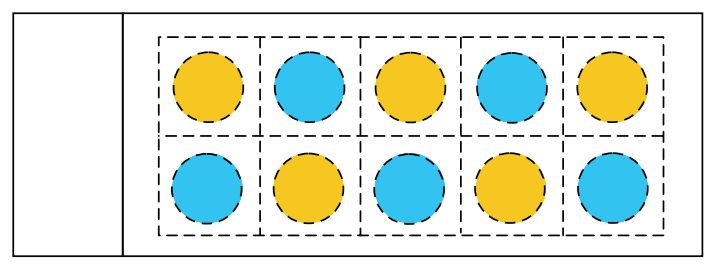

FIgURE 11: Matrix of $2 \times 5$ wells (diameter $=5 \mathrm{~mm}$; distance between wells $=3 \mathrm{~mm}$ ) with CiCOONHS and CouCOONHS grafted alternatively by deposition of $10 \mu \mathrm{L} \approx 60 \mathrm{nmol}$ of each fluorophore solution.

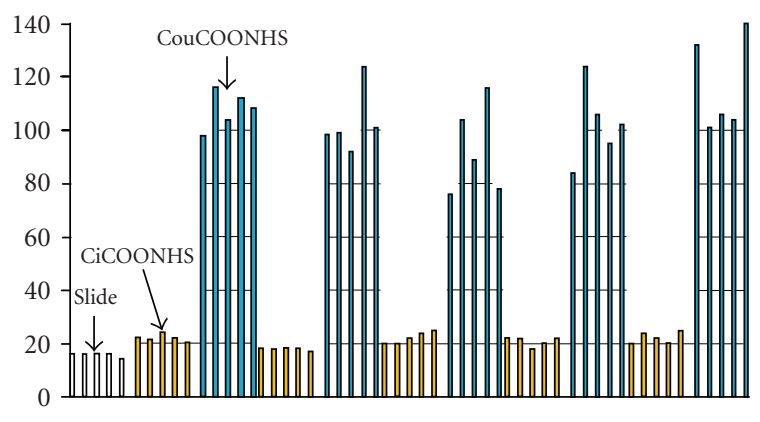

FIgure 12: CiCOONHS and CouCOONHS alternatively grafted in the 10 wells of the matrix analyzed by fluorescence microscopy (filters Zeiss: $\lambda_{\text {exc }}$ [395-440]; $\lambda_{\text {em }}>470 \mathrm{~nm}$; magnification $\times 5$; gauge 4).

area on functionalzed substrates delimited by polymer wells remained reactive as demonstrated by the covalent graftings of suited fluorophores followed by spectrofluorimetry or fluorescence microscopy analyses. We have cured the resin SU8 with a closely spaced hatch pattern until $1 \mathrm{~mm}$ resolution. An over polymerization at the crossing points of the plotting lines has been demonstrated. This problem will be resolved by developing a higher-density microarrays synthesized by photolithographic methods analogous to the techniques used in the semiconductor industry. This original method is based on dynamic photolithographic masks provided by Texas Instrument (Tex, USA) with digital light processor (DLP), often called digital micromirror device (DMD) [11, 12]. Future biomedical implementations include antibody array where different antibodies could be grafted into the wells to realize micro ELISA tests; an immunohistochemistry robot could be used to treat the glass slides.

\section{ACKNOWLEDGMENTS}

This work was supported by the French Research Ministry, MENESR (F. Evenou), CNRS (S. Corbel, F. Baros), and INSERM (M. C. Carré, N. Martinet, M. Donner).

\section{REFERENCES}

[1] J. Hyun and A. Chilkoti, "Micropatterning biological molecules on a polymer surface using elastomeric microwells," 
Journal of the American Chemical Society, vol. 123, no. 28, pp. 6943-6944, 2001.

[2] M. Yan and M. A. Bartlett, "Micro/nanowell arrays fabricated from covalently immobilized polymer thin films on a flat substrate," Nano Letters, vol. 2, no. 4, pp. 275-278, 2002.

[3] D. Falconnet, D. Pasqui, S. Park, et al., "A novel approach to produce protein nanopatterns by combining nanoimprint lithography and molecular self-assembly," Nano Letters, vol. 4, no. 10, pp. 1909-1914, 2004.

[4] J. D. Hoff, L.-J. Cheng, E. Meyhöfer, L. J. Guo, and A. J. Hunt, "Nanoscale protein patterning by imprint lithography," Nano Letters, vol. 4, no. 5, pp. 853-857, 2004.

[5] C. L. Feng, A. Embrechts, G. J. Vancso, and H. Schönherr, "Reactive $\mu \mathrm{CP}$ on ultrathin block copolymer films: localized chemistry for micro- and nano-scale biomolecular patterning," European Polymer Journal, vol. 42, no. 9, pp. 1954-1965, 2006.

[6] M. Furman, S. Corbel, H. Le Gall, O. Zahraa, and M. Bouchy, "Influence of the geometry of a monolithic support on the efficiency of photocatalyst for air cleaning," Chemical Engineering Science, vol. 62, no. 18-20, pp. 5312-5316, 2007.

[7] M. C. Carré, C. Geoffroy-Chapotot, M. Adibnejad, P. Berroy, J. F. Stoltz, and M. L. Viriot, "Fluorescent molecular rotors with specific hydrophilic functions: glucosamine and inositol derivatives," Journal of Fluorescence, vol. 8, no. 1, pp. 53-57, 1998.

[8] C. Muller, P. Even, M.-L. Viriot, and M. C. Carré, "Protection and labelling of thymidine by a fluorescent photolabile group," Helvetica Chimica Acta, vol. 84, no. 12, pp. 3735-3741, 2001.

[9] A. Song, X. Wang, and K. S. Lam, "A convenient synthesis of coumarin-3-carboxylic acids via Knoevenagel condensation of Meldrum's acid with ortho-hydroxyaryl aldehydes or ketones," Tetrahedron Letters, vol. 44, no. 9, pp. 1755-1758, 2003.

[10] G. MacBeath, A. N. Koehler, and S. L. Schreiber, "Printing small molecules as microarrays and detecting protein-ligand interactions en masse," Journal of the American Chemical Society, vol. 121, no. 34, pp. 7967-7968, 1999.

[11] J. Vasco, N. André, and P. Bartolo, "Micro-fabrication: the state-of-the-art," in Proceedings of the 2nd International Conference on Advanced Research in Virtual and Rapid Prototyping, pp. 483-490, Leiria, Portugal, September-October 2005.

[12] J. R. Zyzalo, O. Diegel, and J. Potgieter, "Rapid prototyping: rapid flashing for SLA layering techniques," in Proceedings of the 2nd International Conference on Advanced Research in Virtual and Rapid Prototyping, pp. 449-454, Leiria, Portugal, September-October 2005. 


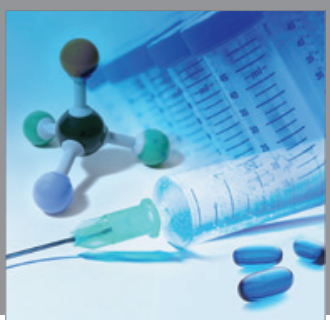

International Journal of

Medicinal Chemistry

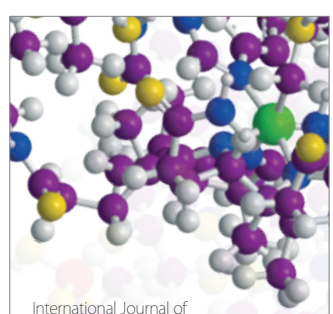

Carbohydrate Chemistry

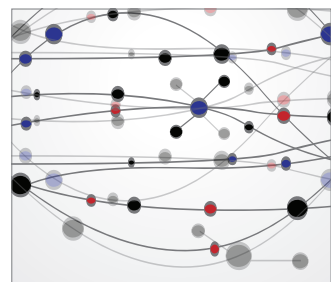

The Scientific World Journal
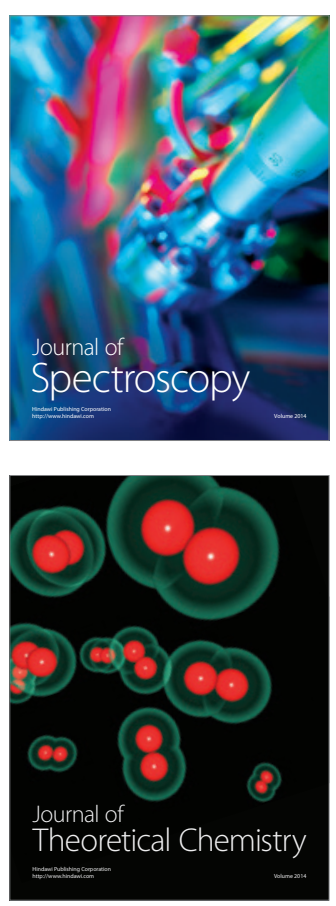
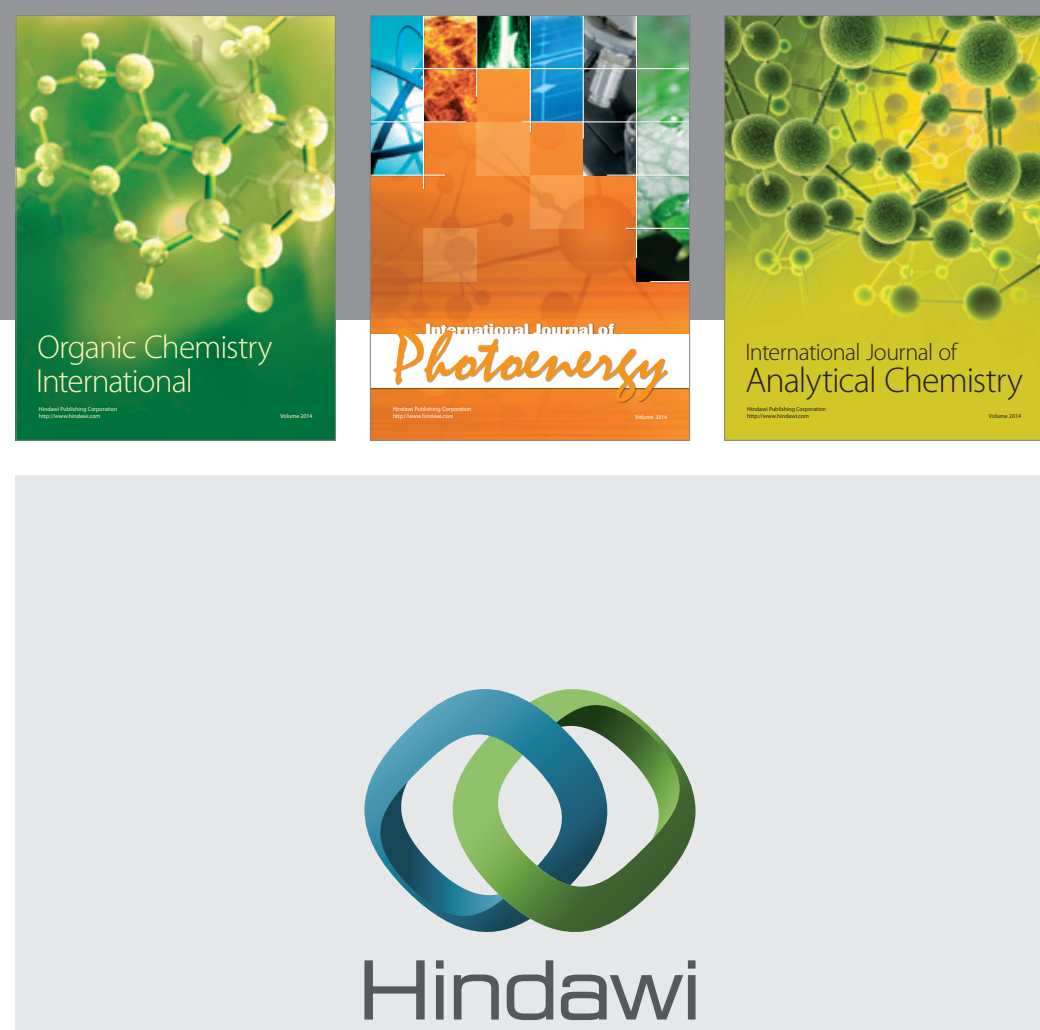

Submit your manuscripts at

http://www.hindawi.com
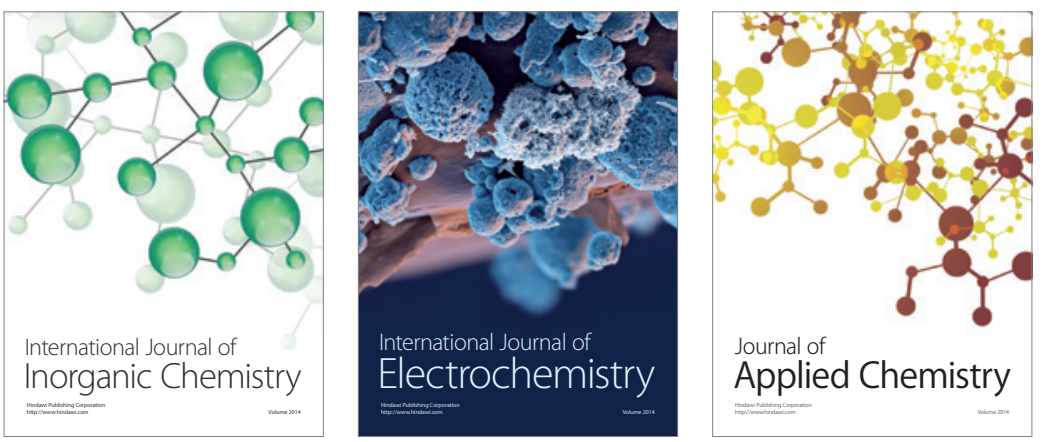

Journal of

Applied Chemistry
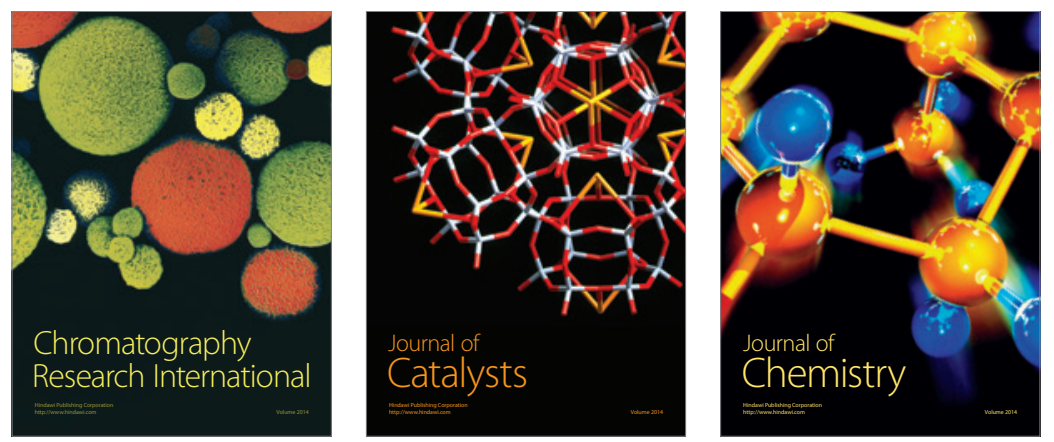
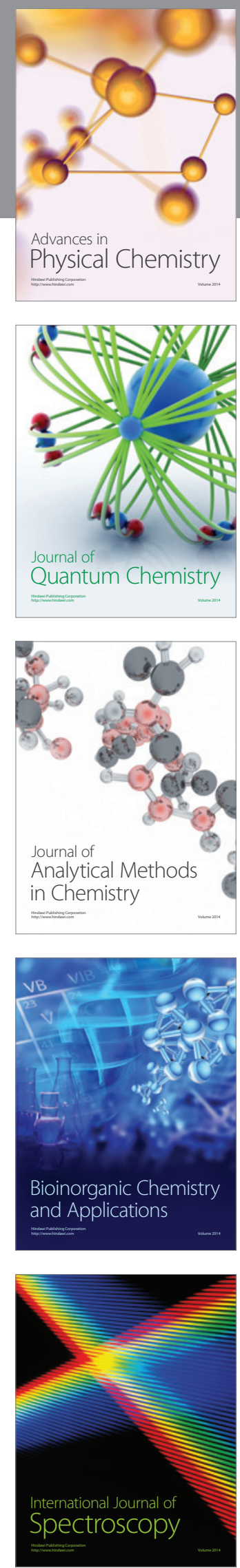